\title{
Understanding herd immunity
}

\author{
C.J.E. Metcalf ${ }^{1,2}$, M. Ferrari ${ }^{3}$, A.L. Graham ${ }^{1}$, B.T. Grenfell ${ }^{1,2}$ \\ ${ }^{1}$ Department of Ecology and Evolutionary Biology, Princeton University, Princeton, \\ USA \\ ${ }^{2}$ Office of Population Research, the Woodrow Wilson School, Princeton University, \\ Princeton, USA \\ ${ }^{3}$ Centre of Infectious Disease Dynamics, the Pennsylvania State University, USA
}

Individual immunity is a powerful force affecting host health and pathogen evolution. Importantly, the effectsof individual immunity also scale up to affect pathogen transmission dynamics and the success of vaccination campaigns for entire host populations. Population-scale immunity is often termed 'herd immunity'.Here, we outline how individual immunity maps to population outcomesand discuss implications for control of infectious diseases. Particular immunologicalcharacteristics may be more or less likely to result in a population level signature of herd immunity, andwe detail this, and alsodiscussother population level outcomes might emerge from individual level immunity.

\section{From individual immunity to herd immunity}

Because of the feedbacks inherent in infectious disease dynamics, individual immunity has a long shadow: infection of one individual simultaneously increases the risk of infection for other individuals in the population by increasing their exposure, butalso reduces the number of susceptible individuals that can be infected because that infected individual will develop individual immunity. For many pathogen life histories, there is a threshold proportion of the population that must be susceptible for the pathogen to successfully spread (Figure 1). Conversely, if theproportion of the population that is immune (by vaccination, or natural infection) exceeds the threshold then incidence of the pathogen will decline. This isknown as the 'herd immunity threshold' and arises because a subset of the susceptible population benefits from 'indirect protection' by the immunization of individuals that surround them (Figure 1).They will be less likely be exposed to infection because most of the individuals that they encounter already have been and are therefore immune, and will not transmit the infection. Indirect protection alters the dynamics of pathogen transmission over the course of an outbreak, and epidemics slow down and then turn over as a result.

For strongly immunizing infections, the key parameter that defines the herd immunity threshold is $\mathrm{R}_{0}$, or the number of new infections generated by the first infectious individual in a completely susceptible population (Figure 1). For measles, for example, an infectious individual who has arrived in a population where no one else has ever experienced measles (or measles vaccine) is expected to infect on average 15 individuals during the infectious phase. In the simplest analysis, the critical proportion of the population that must be immunized to achieve elimination, or $p_{c}$, is defined by $p_{c}=1-1 / \mathrm{R}_{0}$. Thus the more inherently transmissible a pathogen is, the greater the proportion of the population that must be vaccinated to drive it locally extinct. In reality, a unique threshold for immunization associated with pathogen extinction in all populations is unlikely (Fox et al. 1971), as the basic model includes simplifying assumptions, such as homogenous mixing within population (rather than the age-dependent contact rates reported by (Mossong et al. 2008), and through the year (Bjørnstad, Finkenstadt \& Grenfell 2002)), and homogenous vaccination 
coverage (rather than the inequity generally observed (Metcalf et al. 2014)).

Demographic features will also affect the threshold, with higher vaccination coverage required to achieve this threshold in high birth rate contexts(Ferrari, Grenfell \& Strebel 2013). Nevertheless, the concept of herd immunity has provided a powerful benchmark for understanding the relative effort required for control of particular pathogens, and illuminating past successes, such as smallpox elimination. Of course, sterilizing immunity as seen in measles in relatively rare across a diversity of important pathogens. There are a number of other life histories (for example, where immunity may wane, and individuals return to susceptible status) that will generate different population consequences. However, as described below, even quite imperfect immunity following infection can manifest itself via herd immunity at the population level.

\section{Evidence for indirect protection afforded to non-immune individuals}

Since we seldom have detailed observations of chains of transmission (who infected whom), and too rarelyknow the background susceptibility of the people who weren't infected during an outbreak, detecting indirect protection requires careful interpretation of population scale incidence patterns.Data on fluctuations in the number of cases observed within a country or city are frequently available through public health surveillance, and can provide indications of the action of indirect protection. One powerful source of evidence is short-term effects following vaccination introduction, where unvaccinated cohorts show reduced incidence and thus evidence of indirect protection, reviewed in (Fine, Eames \& Heymann 2011). Another source of evidence is changes in cycles of pathogen incidence over the longer term. Susceptible host depletion and replenishment should strongly affect the timing and magnitude of outbreaks where indirect protection is operating (Morris et al. 2015). Accordingly, transition from annual to biennial dynamics was observed for measles after the end of the baby boom (Earn et al. 2000), in line with expectations for this strongly immunizing pathogen: low birth rates mean that susceptibles accumulate more slowly, and they also benefit from indirect protection resulting from a high proportion of immune individuals from the last cycle.For partially immunizing pathogens, indirect protection seems at first less likely. Nevertheless,observed changes in dynamics indicate that even the brief period of reduction in population exposure occasioned by partial immunity could result in indirect protection. In rotavirus, for example, the spatial pattern of timing of epidemics across the USA could be linked to a spatial pattern of birth rates (Pitzer et al. 2011); and furthermore the predicted change from annual to biannual dynamics as a result of vaccination was observed. Similar observations can be made for RSV(Pitzer et al. 2015)and pertussis(Broutin et al. 2010).

\section{From within host to population scale}

Immunity derived from either natural infection or from vaccination can be highly variable, ranging from protection against only clinical disease, or only transmission, or only carriage. In considering how within-host dynamics ofthe immune response to a particular pathogen might shape herd immunity at the population level, arguably, the key aspect is the degree to which acquired immunity limits onward transmission.Imagine a pathogen where immunity considerably reduces symptoms ('disease') for infections occurring after the primary infection, but has no effect on transmission. From the perspective of the pathogen, no individuals have been removed from the population (all the green individuals in Figure 1 would still be 
accessible to infection) - and there is consequently no indirect protection and accordingly, no herd immunity.Some recent work even suggests that disease-onlyblocking vaccines could select for the evolution of increased virulence (Read et al 2015). Again, transmission blocking vaccines provide potentially the safest outcomes (Gandon et al. 2001), which suggests that this should be a key focus in vaccine development to maximize positive impact.

\section{Other intricacies}

It is well known that the interactions of within-host immune processes mean that one pathogen's dynamics may alter the direct protection experienced for other pathogens; this in turn may influence transmission at the population scale. This is particularly clear for immunosuppressive pathogens - there is evidence that HIV can affect acquisition of measles immunity (Moss et al. 2007) and therefore the potential for persistent transmission in populations with high HIV prevalence.Recent results also suggest that infection with measles itself may deplete memory to a range of other pathogens, thus weakening individual immunity and the potential herd-level effects(Mina et al. 2015). Within-host immune interactions might also result in negative indirect effects - via Antibody Dependent Enhancement, infection with one dengue strain can leave individuals more vulnerable to infection with another, but also, potentially, more infectious placing others at higher risk for transmission(Ferguson, Anderson \& Gupta 1999).

\section{Conclusion}

Individual immunity is such a powerful force that its consequences are felt beyond the individual at the population scale. The declineof an epidemic alone is insufficient to conclude the action of indirect protection and herd immunity - changes in transmission linked to behavior changes such as school holidays (Bjørnstad, Finkenstadt \& Grenfell 2002), or fluxes linked to agriculture (Ferrari et al. 2008)can result in such a pattern. However, as described here,changes in epidemiology following interventions such as targeted vaccination, and changes inepidemic cycles in response to vaccination or changes in birth rates can indicate the indirect effect of herd protection. Identifying such signatures is important - indirect protection means that the return on every individual immunized is more than one individual,making immunization a powerful tool in public health.Importantly, these indirect benefits of vaccination reduce population burden of infection, no matter the distance from the herd immunity threshold-and in fact are strongest as the threshold for herd immunity is approached.

\section{Acknowledgements}

This work was funded by the Bill and Melinda Gates Foundation (C.J.E.M., B.T.G., M.F.,), the RAPIDD program of the Science \& Technology Directorate, Department of Homeland Security and the Fogarty International Center, National Institutes of Health (C.J.E.M., B.T.G., M.F., A.L.G.).

\section{References}

Bjørnstad, O.N., Finkenstadt, B. \& Grenfell, B.T. (2002) Endemic and epidemic dynamics of measles: Estimating epidemiological scaling with a time series SIR model. Ecological Monographs, 72, 169-184.

Broutin, H., Viboud, C., Grenfell, B., Miller, M. \& Rohani, P. (2010) Impact of vaccination and birth rate on the epidemiology of pertussis: a 
comparative study in 64 countries. Proceedings of the Royal Society of London B: Biological Sciences, 277, 3239-3245.

Earn, D.J.D., Rohani, P., Bolker, B.M. \& Grenfell, B.T. (2000) A simple model for complex dynamical transitions in epidemics. Nature,287, 667-670.

Ferguson, N., Anderson, R. \& Gupta, S. (1999) The effect of antibody-dependent enhancement on the transmission dynamics and persistence of multiplestrain pathogens. Proceedings of the National Academy of Sciences,96, 790-794.

Ferrari, M.J., Grais, R.F., Bharti, N., Conlan, A.J.K., Bjørnstad, O.N., Wolfson, L.J., Guerin, P.J., Djibo, A. \& Grenfell, B.T. (2008) The dynamics of measles in sub-Saharan Africa. Nature,451, 679-684.

Ferrari, M.J., Grenfell, B.T. \& Strebel, P. (2013) Think globally, act locally: the role of local demographics and vaccination coverage in the dynamic response of measles infection to control. Philosophical Transactions of the Royal Society,368, 2012014.

Fine, P., Eames, K. \& Heymann, D.L. (2011) "Herd immunity": a rough guide. Clinical Infectious Diseases, 52, 911-916.

Fox, J.P., Elveback, L., Scott, W., Gatewood, L. \& Ackerman, E. (1971) Herd immunity: basic concept and relevance to public health immunization practices. American Journal of Epidemiology, 94, 179-189.

Gandon, S., Mackinnon, M.J., Nee, S. \& Read, A.F. (2001) Imperfect vaccines and the evolutoin of pathogen virulence Nature,414, 751-756.

Metcalf, C.J.E., Tatem, A., Bjornstad, O.N., Lessler, J., O’Reilly, K., Takahashi, S., Cutts, F.T. \& Grenfell, B.T. (2014) Transport networks and inequities in vaccination: remoteness shapes measles vaccine coverage and prospects for elimination across Africa. Epidemiology and Infection, 1-10.

Mina, M.J., Metcalf, C.J.E., de Swart, R.L., Osterhaus, A. \& Grenfell, B.T. (2015) Long-term measles-induced immunomodulation increases overall childhood infectious disease mortality. Science,348, 694-699.

Morris, S.E., Pitzer, V.E., Viboud, C., Metcalf, C.J.E., Bjørnstad, O.N. \& Grenfell, B.T. (2015) Demographic buffering: titrating the effects of birth rate and imperfect immunity on epidemic dynamics. Journal of the Royal Society Interface,12, 20141245.

Moss, W.J., Scott, S., Mugala, N., Ndhlovu, Z., Beeler, J.A., Audet, S.A., Ngala, M., Mwangala, S., Nkonga-Mwangilwa, C. \& Ryon, J.J. (2007) Immunogenicity of standard-titer measles vaccine in HIV-1-infected and uninfected Zambian children: an observational study. Journal of Infectious Diseases, 196, 347-355.

Mossong, J., Hens, N., Jit, M., Beutels, P., Aranen, K., Mikolajczyk, R., Massari, M., Salmaso, S., Scalia Tomba, G., Wallinga, J., Heijne, J., Sadkowska-Todys, M., Rosinska, M. \& Edmunds, W.J. (2008) Social Contacts and Mixing Patterns Relevant to the Spread of Infectious Diseases. PloS Medicine,5, e74.

Pitzer, V.E., Patel, M.M., Lopman, B.A., Viboud, C., Parashar, U.D. \& Grenfell, B.T. (2011) Modeling rotavirus strain dynamics in developed countries to understand the potential impact of vaccination on genotype distributions. Proceedings of the National Academy of Sciences,108, 19353-19358.

Pitzer, V.E., Viboud, C., Alonso, W.J., Wilcox, T., Metcalf, C.J., Steiner, C.A., Haynes, A.K. \& Grenfell, B.T. (2015) Environmental drivers of the spatiotemporal 
dynamics of respiratory syncytial virus in the United States. PLoS Pathogens, 11, e1004591. 
Figure 1: Herd immunity in a closed population (flow diagram on the left, no new susceptibles are born, and no one leaves or enters) for a completely immunizing infection with $R_{0}=5$. The top panel shows $R_{E}$, the effective reproduction number, reflecting the number of new infected individuals per one infectious individual. $R_{E}$ is defined by the product of $\mathrm{R}_{0}$ and the proportion of the population that is susceptible. The bottom panel shows the proportion of individuals that are susceptible, infected, and recovered. Initially, everyone is susceptible (black line), and an infected individual is introduced. The infection spreads rapidly (red line) but reaches a threshold where new infected individuals can infect less than 1 susceptible individual $\left(\mathrm{R}_{\mathrm{E}}<1\right.$, top panel) as most of the population is now recovered and immune (green line). The few remaining susceptible individuals benefit from 'indirect protection'. These dynamics are illustrated in the schema below, where circles indicate individuals, colours indicate whether they are susceptible, infected, or recovered; arrows indicate successful transmission, and blocked arrows show individuals protected through their own immunity; indirectly protected individuals are also indicated. The herd immunity threshold is the fraction of the population that is susceptible at the point where $\mathrm{R}_{\mathrm{E}}$ falls below 1 , and the number of infectious individuals peaks, indicated by the dotted vertical line. Mathematically, this equates to $\mathrm{p}_{\mathrm{c}}=1-1 / \mathrm{R}_{0}=0.8$. 

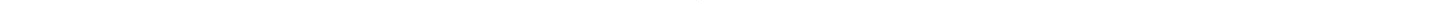